\title{
Hypoplasia Resulting from Nutritional Deficiency: A Case Report
}

\author{
Sarah A Mubaraki
}

\begin{abstract}
Aim: The aim of the study was provide information to dentists about the causes of enamel hypoplasia and treatment alternatives available to dentists working with immature permanent teeth.

Background: Hypoplasia resulting from nutritional deficiency is a developmental anomaly resulting from defects in amelogenesis. The treatment strategy should be based on the degree of involvement as well as the functional and esthetic needs in each case.

Case description: This article reports the case of a 14-year-old boy presenting with enamel hypoplasia affecting multiple anterior and posterior teeth that were treated conservatively.

Conclusion: Enamel hypoplasia resulting from severe calcium deficiency affects esthetics, child self-esteem, and quality of life. This report highlights the history of severe calcium deficiency during childhood, and conservative treatment to improve the esthetic and reduce tooth sensitivity.

Clinical significance: Conservative approach was done for all the affected permanent teeth and was used to improve the esthetic and to have an effective transitional treatment to tell the child to reach a stage of stable occlusion.

Keywords: Enamel hypoplasia, Nutritional deficiency, Young permanent teeth.

International Journal of Clinical Pediatric Dentistry (2019): 10.5005/jp-journals-10005-1700
\end{abstract}

\section{BACKGROUND}

Enamel hypomineralization occurs frequently in children worldwide and can lead to the development of caries, occlusion abnormalities, esthetic issues, ${ }^{1}$ and decreased quality of life. ${ }^{2}$ Enamel hypoplasia is defined as a qualitative visible defect of enamel and is histomorphological identified as an external defect involving the surface of the enamel associated with reduced enamel thickness. ${ }^{3}$

Enamel hypoplasia is the result of a disruption in the process of enamel matrix formation causing defects in the quality and thickness of the enamel. ${ }^{4}$ Enamel hypoplasia may be mild and may result in pitting of the enamel surface or the development of a horizontal line across the enamel of the crown. If ameloblastic activity has been disrupted for a long period, gross areas of irregular or imperfect enamel formation occur. Postnatal amelogenesis is confined to the portion of the crown located cervically from the enamel area present at birth.

Hypoplasia is often associated with systemic disturbances that occur during the development of the permanent teeth, including exanthematous fevers. However, deficiency states, particularly those related to deficiencies in vitamins $A, C$, and D, calcium, and phosphorus, have also been shown to cause enamel hypoplasia. ${ }^{5,6}$

Clinical management of hypoplasia is difficult due to the rapid development of dental caries, limited cooperation of children, and repeated breakdown of restorations.

\section{Case Description}

A 14-year-old boy presented to the Division of Pedodontics of Preventive Dentistry Department, Riyadh Elm University, Riyadh, Kingdom of Saudi Arabia, with a complaint of esthetic defects and sensitivity of multiple teeth. His prenatal, birth, medical, and family histories were unremarkable. His mother reported that her son had
Preventive Dentistry Department, Pedodontic Division, College of Dentistry, Riyadh Elm University, Riyadh, Kingdom of Saudi Arabia

Corresponding Author: Sarah A Mubaraki, Preventive Dentistry Department, Pedodontic Division, College of Dentistry, Riyadh Elm University, Riyadh, Kingdom of Saudi Arabia, Phone: +966 542305554, e-mail: sarah.mubaraki@riyadh.edu.sa

How to cite this article: Mubaraki SA. Hypoplasia Resulting from Nutritional Deficiency: A Case Report. Int J Clin Pediatr Dent 2019;12(6):573-576.

Source of support: Nil

Conflict of interest: None

a severe calcium deficiency from birth up to 6 months of age, and he was admitted to the hospital for 2 months for treatment.

Clinical examination revealed that enamel hypoplasia affected the maxillary central incisors, maxillary canines, mandibular incisors and canines, and all maxillary and mandibular first permanent molars (Fig. 1). Full orthodontic assessment showed typical adenoid facies; increased facial height; maxillary arch constriction; anterior open bite; moderate crowding in the maxillary and mandibular arches (upper: $-4.5 \mathrm{~mm}$, lower: $-6 \mathrm{~mm}$ ); rotation of the maxillary anterior teeth; and class II mandibular, molar, and canine relationship bilaterally. Cephalometric analysis was used for treatment planning (Figs 2 and 3).

Radiographically, very thin enamel and dentin layers were observed (Fig. 4). A diagnosis of hypoplasia resulting from nutritional deficiency was made based on these findings.

Since the appearance of the involved teeth affected the child psychologically, a composite resin restoration was performed for all affected teeth, and stainless steel crowns were placed on all permanent molars. A comprehensive orthodontic treatment was required (Fig. 5). 

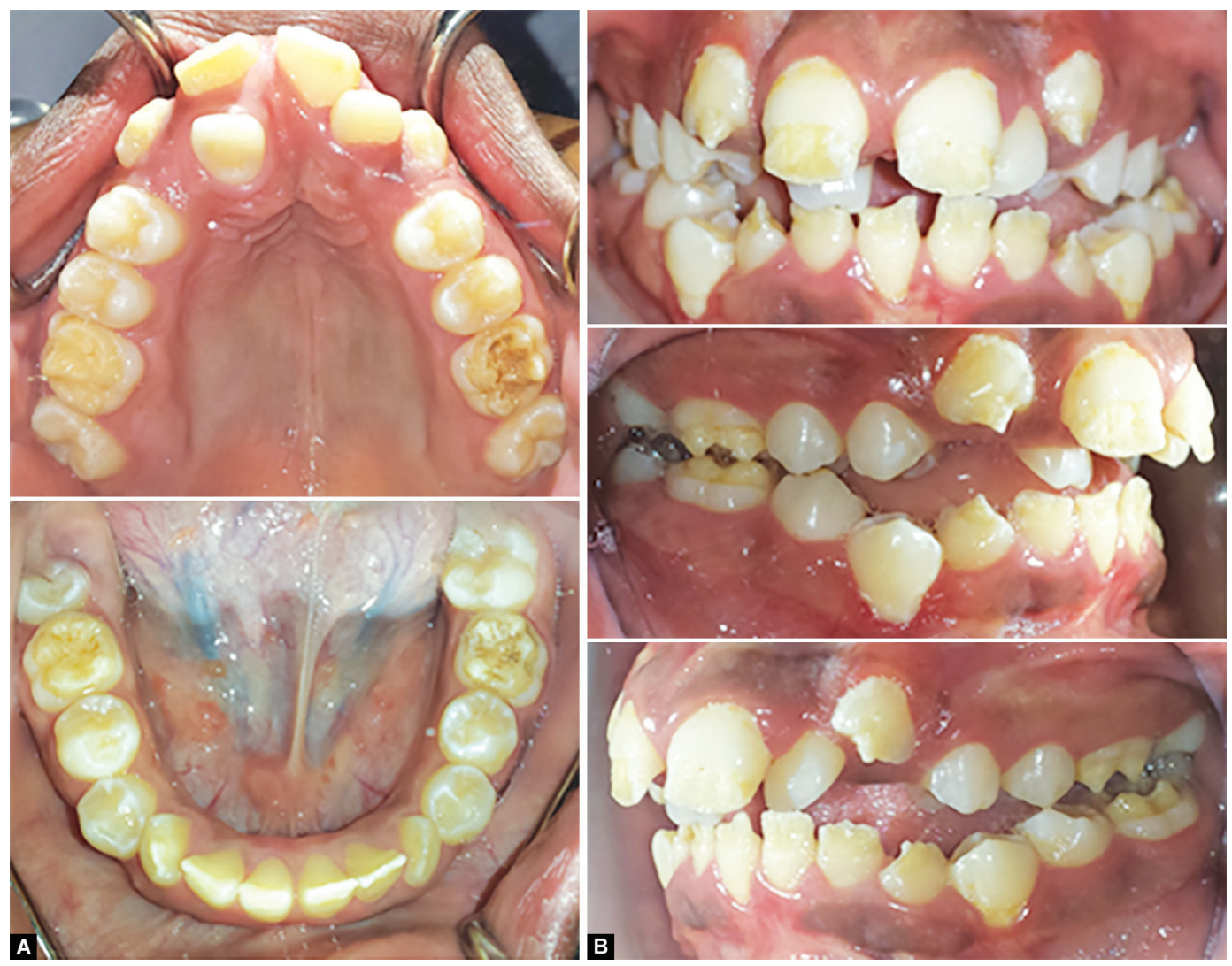

Figa 1A and B: Enamel hypoplasia occurred during early childhood. Enamel formation took place on the incisal third of the maxillary central incisors, maxillary canines, mandibular incisors and canines, and all the first permanent molars. The maxillary lateral incisors are normal

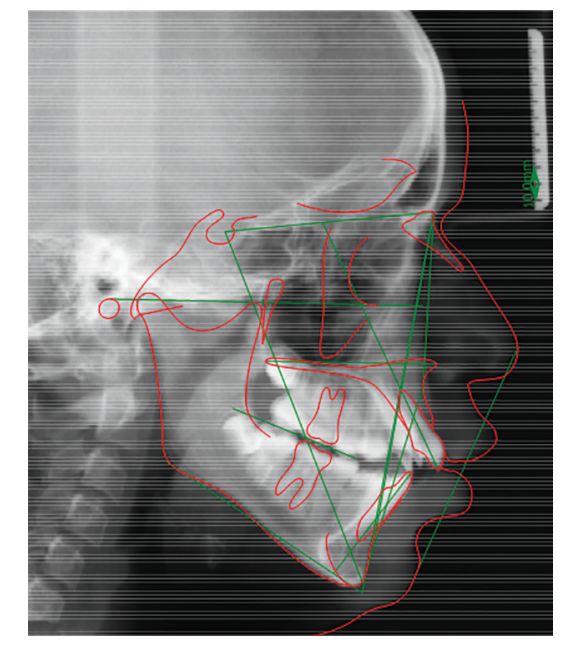

Fig. 2: Cephalometric radiograph

The patient will be continued to be seen regularly for follow-up to the age of 19 years for final crowns and onlays to be fabricated.

\section{Discussion}

In this study, a case of enamel hypoplasia resulting from severe calcium deficiency was reported. The structure of the teeth is influenced by nutrition during tooth development, and such defects occur due to the ameloblasts' sensitivity to environmental changes, leading to distinctive bands of malformed enamel. ${ }^{7}$
Nutritional deficiencies affect the structure of teeth and increase the risk of development of dental caries. ${ }^{8}$ A conservative treatment should be applied to preserve the affected teeth for as long as possible. Placement of stainless steel crowns on all the first permanent molars required minimal tooth preparation and will allow the passive eruption of the teeth and normal jaw development. All affected anterior teeth were restored by composite resin restoration to improve esthetics and reduce tooth sensitivity.

In the present case, the child and his mother were satisfied with the treatment, but the rehabilitation cannot be considered ideal because the patient's oral esthetic and function were not completely restored. At present, the patient is under controlled visits to observe the growth and development of the maxilla and mandible. However, the prognosis of the affected permanent teeth is good.

\section{Conclusion}

Enamel hypoplasia resulting from severe calcium deficiency affects esthetics, child self-esteem, and quality of life. This report highlights the history of severe calcium deficiency during childhood, and conservative treatment to improve the esthetic and reduce tooth sensitivity.

\section{Clinical Significance}

Minimal tooth reduction was done for all the affected permanent teeth to improve the esthetic, which was the main complaint of the 
Hypoplasia Resulting from Nutritional Deficiency: A Case Report

\begin{tabular}{|c|c|c|c|c|c|c|c|c|c|c|c|c|c|}
\hline & \multicolumn{13}{|c|}{ Riyadh } \\
\hline Variable & Description & Norm [] & Value [] & Diff & \multicolumn{9}{|c|}{ Deviation } \\
\hline \multicolumn{14}{|l|}{ Skeletal } \\
\hline $\mathrm{FH}-\mathrm{SN}\left({ }^{\circ}\right)$ & Frankfurt horizontal to ant.crania & $6.0 \pm 4^{\circ}$ & $6.5^{\circ}$ & 0.0 & & 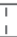 & 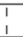 & & $\begin{array}{l}1 \\
1\end{array}$ & $\begin{array}{c}1 \\
1\end{array}$ & $\begin{array}{l}1 \\
\vdots\end{array}$ & $\begin{array}{c}1 \\
1\end{array}$ & ! \\
\hline A-P & & & & & & 1 & 1 & & 10 & 1 & 1 & 1 & 1 \\
\hline FH-NPog $\left({ }^{\circ}\right)$ & Facial angle & $88.3 \pm 6.4^{\circ}$ & $80.6^{\circ}$ & -1.3 & & 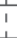 & 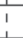 & & $\begin{array}{ll}1 \\
1\end{array}$ & 1 & 1 & 1 & 1 \\
\hline N-A-Pog $\left({ }^{\circ}\right)$ & Convexity & $5.0 \pm 9^{\circ}$ & $13.5^{\circ}$ & 0.0 & & 1 & 1 & & 1 & 1 & 1 & 1 & 1 \\
\hline SNA $\left({ }^{\circ}\right)$ & SNA angle & $81.0 \pm 2.7^{\circ}$ & $81.2^{\circ}$ & -0.0 & 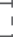 & + & + & ( & 10 & 1 & 1 & 1 & 1 \\
\hline SNB $\left({ }^{\circ}\right)$ & SNB angle & $78.0 \pm 2^{\circ}$ & $74.4^{\circ}$ & -1.6 & & 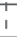 & i & & Q i & i & i & i & i \\
\hline ANB $\left({ }^{\circ}\right)$ & ANB angle & $3.0 \pm 2^{\circ}$ & $6.8^{\circ}$ & +1.8 & & 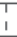 & & & $\frac{1}{1}$ & 1 & 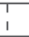 & 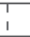 & i \\
\hline AB-Npog $\left({ }^{\circ}\right)$ & Angle of $A-B$ to N-Pog & $-6.1 \pm 6.6^{\circ}$ & $-7.2^{\circ}$ & 0.0 & & $\begin{array}{l}1 \\
1\end{array}$ & 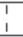 & & $\begin{array}{l}1 \\
1 \\
\end{array}$ & $\gamma_{1}$ & $\begin{array}{l}1 \\
1\end{array}$ & $\begin{array}{l}1 \\
1 \\
\end{array}$ & $\begin{array}{c}1 \\
1 \\
\end{array}$ \\
\hline A-N Perp. (mm) & Distance of A-Point to N-Perp. & $0.0 \pm 2.8 \mathrm{~mm}$ & $-2.1 \mathrm{~mm}$ & 0.0 & tis & i & 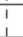 & & 1 & 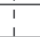 & i & 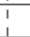 & i \\
\hline Pog-N Prep. (mm) & Distance of pogonion to N-Perp. & $-5.0 \pm 3 \mathrm{~mm}$ & $-18.0 \mathrm{~mm}$ & -10.0 & the & 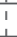 & 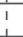 & & 1 & 1 & 1 & 1 & 1 \\
\hline Cond-A (mm) & Midface length & $78.9 \pm 4 \mathrm{~mm}$ & $88.0 \mathrm{~mm}$ & +5.1 & & 蒋 & 1 & 1 & 1 & 1 & 1 & 1 & 1 \\
\hline Cond-Gn (mm) & Mandibular length & $93.6 \pm 4 \mathrm{~mm}$ & $108.6 \mathrm{~mm}+$ & 11.0 & & 1 & 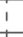 & 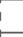 & 1 & 4 & 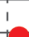 & 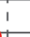 & 1 \\
\hline Wits $(\mathrm{mm})$ & Wits appraisal & $-1.0 \pm 2 \mathrm{~mm}$ & $-3.6 \mathrm{~mm}$ & -0.6 & 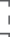 & 1 & 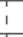 & & 1 & 1 & 1 & 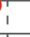 & I \\
\hline Vertical & & & & & & 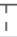 & T & & $\begin{array}{ll}1 \\
1 & 1\end{array}$ & i & T & 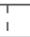 & i \\
\hline $\mathrm{PP}-\mathrm{MP}\left({ }^{\circ}\right)$ & Palatal plane to mand. plane & $26.0^{\circ}$ & $33.0^{\circ}$ & +7.0 & 7 & $\begin{array}{l}1 \\
\vdots\end{array}$ & $\begin{array}{l}1 \\
1\end{array}$ & & $\begin{array}{ll} & 1 \\
1 & 1\end{array}$ & $\frac{1}{1}$ & $\begin{array}{l}1 \\
1\end{array}$ & $\begin{array}{c}1 \\
\end{array}$ & $\begin{array}{c}1 \\
1\end{array}$ \\
\hline FMA $\left({ }^{\circ}\right)$ & Frankfurt horizontal to mand. plane & $22.4 \pm 9.8^{\circ}$ & $32.5^{\circ}$ & +0.3 & & 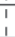 & 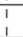 & 1 & i & 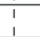 & 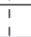 & 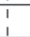 & 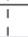 \\
\hline $\mathrm{SGn}-\mathrm{FH}\left({ }^{\circ}\right)$ & Y-Axis - Downs & $66.0 \pm 7^{\circ}$ & $67.3^{\circ}$ & 0.0 & & 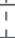 & 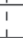 & & 1 & $\frac{1}{1}$ & 1 & 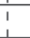 & 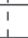 \\
\hline ANS - Me (mm) & Lower face height & $65.0 \pm 4.5 \mathrm{~mm}$ & $71.8 \mathrm{~mm}$ & +2.3 & & 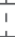 & 1 & I & 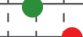 & 1 & 1 & 1 & 1 \\
\hline ANS-Me / N-Me (\%) & Lower to total facial height & $55.0 \pm 3 \%$ & $62.2 \%$ & +4.2 & t & 1 & I & & 1 & 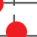 & 1 & 1 & 1 \\
\hline Dental & & & & & & + & i & & 1 & 1 & i & i & I \\
\hline A-P & & & & & & 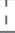 & T & ז & 1 & i & 1 & i & i \\
\hline $\mathrm{U} 1-\mathrm{PP}\left({ }^{\circ}\right)$ & Upper incisor to palatal plane & $110.0^{\circ}$ & $1159^{\circ}$ & +5.9 & 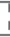 & $\begin{array}{l}1 \\
1\end{array}$ & $\begin{array}{l}1 \\
1\end{array}$ & & $\begin{array}{ll}1 \\
1 & 1 \\
\end{array}$ & $\begin{array}{c}1 \\
1\end{array}$ & $\begin{array}{l}1 \\
1\end{array}$ & $\begin{array}{c}1 \\
\end{array}$ & $\begin{array}{c}1 \\
1\end{array}$ \\
\hline U1-SN $\left({ }^{\circ}\right)$ & Upper incisor to ant. cranial base & $105.0 \pm 9^{\circ}$ & $109.8^{\circ}$ & 0.0 & & $\begin{array}{l}1 \\
1\end{array}$ & $\begin{array}{l}1 \\
1\end{array}$ & & 111 & $\begin{array}{l}1 \\
1\end{array}$ & $\begin{array}{l}1 \\
1\end{array}$ & $\begin{array}{l}1 \\
1\end{array}$ & $\begin{array}{l}1 \\
1\end{array}$ \\
\hline U1-A-Pog (mm) & Upper incisor to A-Pog & $5.0 \pm 5.4 \mathrm{~mm}$ & $11.7 \mathrm{~mm}$ & +1.3 & & i & i & 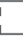 & 1 & i & i & 1 & i \\
\hline $\mathrm{U} 1-\mathrm{NA}(\mathrm{mm})$ & Upper incisor to N-A & $4.3 \pm 2.7 \mathrm{~mm}$ & $6.1 \mathrm{~mm}$ & 0.0 & & 1 & 1 & & 11 & 1 & 1 & 1 & 1 \\
\hline & & & & & & i & i & & 11 & 1 & 1 & I & 1 \\
\hline L1-MP $\left({ }^{\circ}\right)$ & Lower incisor to mand. plane & $(-90) 4.0 \pm 6^{\circ}$ & $3.8^{\circ}$ & 0.0 & & 1 & 1 & & $\begin{array}{ll}1 \\
1 \\
1 & 1 \\
\end{array}$ & i & i & + & i \\
\hline L1-APog (mm) & Lower incisor to A-Pog & $1.3 \pm 4.7 \mathrm{~mm}$ & $4.2 \mathrm{~mm}$ & 0.0 & & 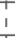 & i & & 101 & i & i & i & i \\
\hline L1-NB (mm) & Lower incisor to N-B & $6.0 \pm 1.8 \mathrm{~mm}$ & $7.4 \mathrm{~mm}$ & 0.0 & & T & 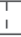 & & $\begin{array}{ll}1 & 1 \\
1 & 1\end{array}$ & 1 & 1 & 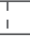 & $\begin{array}{c}1 \\
1\end{array}$ \\
\hline & & & & & & $\begin{array}{l}1 \\
1\end{array}$ & $\begin{array}{c}1 \\
1\end{array}$ & & $\begin{array}{lll} & 1 & 1 \\
1 & 1 & 1 \\
\end{array}$ & $\begin{array}{l}1 \\
1 \\
\end{array}$ & $\begin{array}{l}1 \\
1\end{array}$ & $\frac{1}{1}$ & $\begin{array}{l}1 \\
1 \\
\end{array}$ \\
\hline U1-L1 ( $\left.{ }^{\circ}\right)$ & Interincisal angle & $126.0 \pm 21.7^{\circ}$ & $117.3^{\circ}$ & 0.0 & & 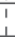 & i & 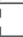 & 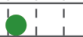 & 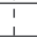 & i & 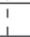 & 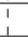 \\
\hline Soft tissue & & & & & & $\frac{1}{1}$ & 1 & 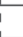 & 1 & 1 & 1 & 1 & 1 \\
\hline Cotg-Sn-Ls $\left(^{\circ}\right)$ & Nasolabial angle & $102.0 \pm 8^{\circ}$ & $78.1^{\circ}$ & -15.9 & & & $y$ & 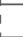 & 11 & 1 & 1 & 1 & 1 \\
\hline LL-E plane (mm) & Lower lip to esthetic plane & $-2.0 \pm 2 \mathrm{~mm}$ & $6.3 \mathrm{~mm}$ & +6.3 & & + & 1 & & 1 & 1 & + & + & 1 \\
\hline UL-E plane (mm) & Upper lip to esthetic plane & $-4.0 \pm 2 \mathrm{~mm}$ & $5.6 \mathrm{~mm}$ & +7.6 & & i & 1 & i & 111 & 1 & 1 & ic & 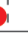 \\
\hline
\end{tabular}

Fig. 3: Cephalometric analysis outcome
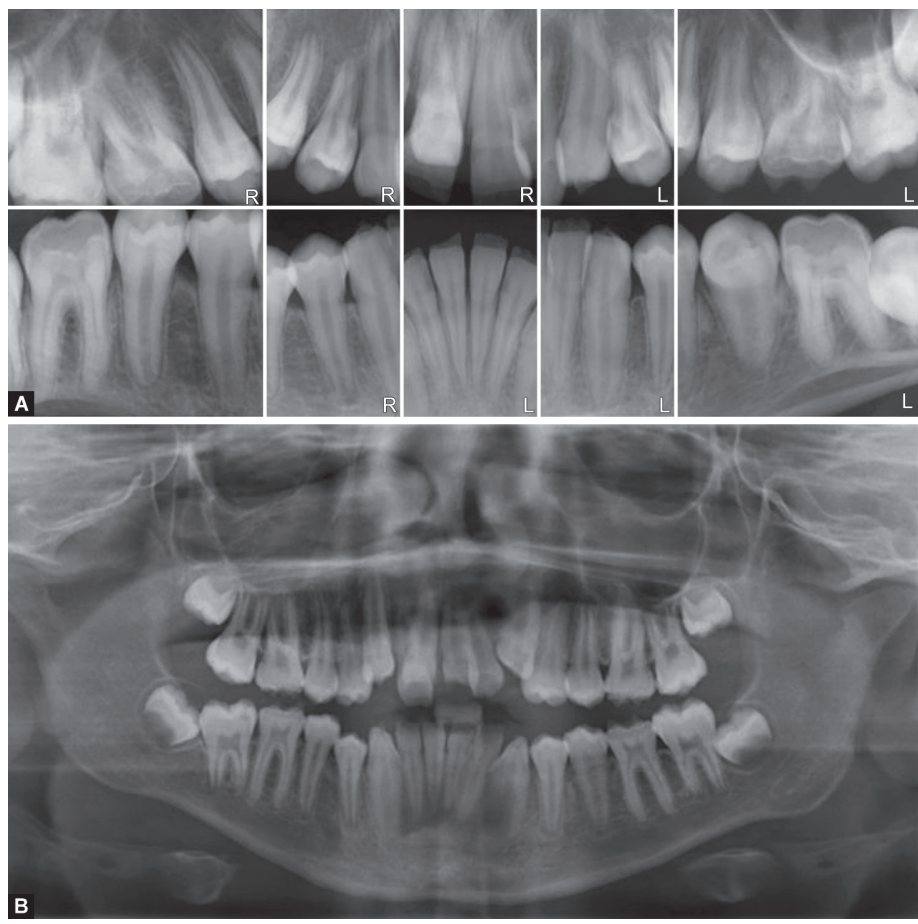

Figs 4A and B: Radiographically, the maxillary central incisors, maxillary canines, mandibular incisors and canines, and all first permanent molars showed very thin enamel and dentin layers. The maxillary lateral incisors are normal 

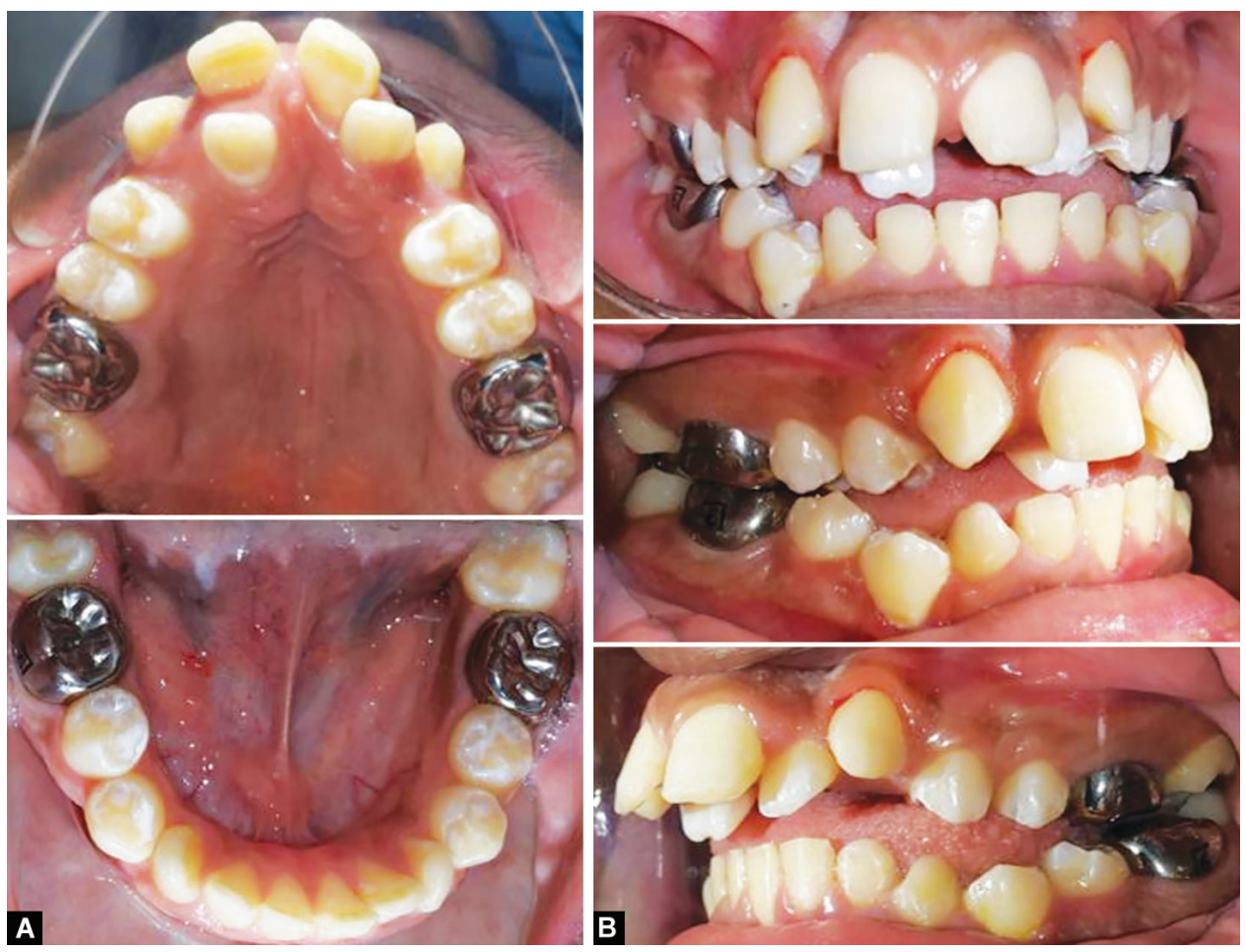

Figs 5A and B: Posttreatment photographs; a composite resin restoration was applied to all affected anterior teeth, and stainless steel crowns were applied to all the first permanent molars

child, as he is only 14 years old, this treatment serves as transitional treatment until he reaches a stage of stable occlusion, then he can go for a more comprehensive approach.

\section{Acknowledgment}

We are grateful for the cooperation of the patient and his family.

\section{References}

1. Weerheijm KL. Molar incisor hypomineralization (MIH). Eur J Paediatr Dent 2003;4(3):114-120.

2. Portella PD, Menoncin BLV, de Souza JF, et al. Impact of molar-incisor hypomineralization on quality of life in children with early mixed dentition: a hierarchical approach. Int J Paediatr Dent 2019;29(4): 496-506. DOI: 10.1111/ipd.12482.

3. Jalevik B, Noren JG. Enamel hypomineralization of permanent first molars: a morphological study and survey of possible etiological factors. Int J Paediatr Dent 2000;10(4):278-289. DOI: 10.1046/j.1365263x.2000.00210.x.

4. Priya PG, John JB, Elango I. Turner's hypoplasia and non-vitality: a case report of sequelae in permanent tooth. Contemp Clin Dent 2010;1(4):251-254.

5. Masterson EE, Fitzpatrick AL, Enquobahrie DA, et al. Malnutritionrelated early childhood exposures and enamel defects in the permanent dentition: a longitudinal study from the bolivian amazon. Am J Phys Anthropol 2017;164(2):416-423. DOI: 10.1002/ajpa.23283.

6. Geber J. Skeletal manifestations of stress in child victims of the great irish famine (1845-1852): prevalence of enamel hypoplasia, Harris lines, and growth retardation. Am J Phys Anthropol 2014;155(1): 149-161. DOI: 10.1002/ajpa.22567.

7. Nanci A, Ten Cate AR. Oral histology, development, structure and function; 2008. p. 400.

8. Rugg-Gunn AJ. Nutrition, diet and dental public health. Community Dent Health 1993;10(Suppl 2):47-56. 